\title{
Testing 3-D Reconstruction Methods for Electron Tomography
}

\author{
S. Mehraeen, ${ }^{*}$ H. Stahlberg, ${ }^{* *}$ J. E. Evans, ${ }^{* *}$ C. Meesters, ${ }^{* *}$ I. Arslan, ${ }^{* * *}$ P. A. Midgley, ${ }^{* * *}$ and \\ N. D. Browning*,**** \\ * Department of Chemical Engineering \& Materials Science, University of California, Davis, One \\ Shields Avenue, Davis, CA 95616 \\ ** Department of Molecular \& Cellular Biology, University of California, Davis, One Shields \\ Avenue, Davis, CA 95616 \\ *** Department of Materials Science and Metallurgy, University of Cambridge, Pembroke Street, \\ Cambridge CB2 3QZ, UK \\ **** National Center for Electron Microscopy, Lawrence Berkeley National Laboratory, One \\ Cyclotron Road, Berkeley, CA 94720
}

The technique of 3-dimensional imaging known as "electron tomography", while not new in the field of biological sciences, has rarely been used for Materials Science. Electron tomography refers to any mathematical process used to reconstruct the 3-dimensional structure of a specimen from a series of 2-dimensional projections of that structure [1]. A variety of software packages are used to perform different functions in this mathematical process. However, so far no consensus method has been determined to provide structural information in 3 dimensions with the $0.1-20 \mathrm{~nm}$ spatial resolution required for many Materials Science applications. As developing nanostructural designs continues, interpretation of these structures merely based on 2-dimensinal electron microscopic projections of such materials and their complex overlapping morphologies becomes a less authentic practice and a routine method for reconstructing the 3-D structure of materials must be developed.

In the present research, a 3D structure of bimetallic $\mathrm{Sn}_{\mathrm{x}} \mathrm{Si}_{1-\mathrm{x}}$ quantum dots in a mesoporous silica matrix was reconstructed using the IMOD and SPIDER software packages responsible for alignment, re-construction and characterization of the 3-dimensional volume. IMOD contains tools for assembling and aligning data within multiple sizes of image stacks, viewing 3-D data from any orientation, modeling and display of the image files [2]. The software reconstructs the 3-D structure of the sample from a series of projection images recorded at different sample tilt. Contrasting spots in the projections are employed as markers to guide the alignment of the projection images with respect to each other. Because biological samples usually lack sufficiently contrasted particles, gold particles in controlled size and quantity are spread on these samples before taking the image. In our case the necessary contrast was provided by taking the images using high-angle annular dark field scanning transmission electron microscopy (HAADF STEM) technique.

In the contrast, the high-Z (atomic number) particles of intermetallic Sn quantum dots in the lower-Z silicon matrix were seen as bright individual clusters in a dark matrix. A series of 149 HAADF STEM projections of the specimen which were recorded at a tilt range of $-74^{\circ}$ to $+74^{\circ}$ on a "FEI 200KV field-emission Tecnai" at the University of Cambridge, processed with IMOD. The software was used to track a selected set of markers through the 149 stacks to align them with respect of each other and yield a 3-D image, which then can be viewed along any axis. It appears that the performance of the IMOD software is a function of the quantity and distance of the markers; more markers spaced uniformly across the image produce a better final volume whereas fewer markers or markers spaced very close to each other are likely to yield reconstructions of lower resolution. The 
tracking process of the chosen markers by computed correlation methods, involved much more human intervention than typical for biological samples and resulted in a significantly improved resolution. The final result was a 3-dimensional volume shown in figure 1A. The reconstructed volume can be visualized as slices through sequential layers and as such can reveal detailed information as to the structure of the individual quantum dots. However, the 3-D reconstruction suffered inevitably from the limited range of tilt angles of the STEM images. The closer the tilt angle range to the ideal situation $\left(-90^{\circ}\right.$ to $\left.+90^{\circ}\right)$, the smaller the so-called missing wedge of information in the Fourier -space representation of the reconstruction, which leads to a reduced resolution in the vertical direction. The 3D reconstruction of the specimen revealed the location, shape and distribution of quantum dots sitting on the top and bottom surfaces of the silicon matrix.

Finally the resulting 3-D reconstruction volume was analyzed to determine the accurate size, crystal shape, orientation, and size distribution of the individual quantum dots using "SPIDER" - a modular image processing software suite containing operations to transform and display images and volumes. SPIDER provided a programming environment, which allowed for breaking the initial volume into smaller modules according to specific practical considerations - primarily containing a single quantum dot [3]. The resulting modules were then analyzed to produce the desired quantitative information including size, shape, dimensions, and symmetry of the individual quantum dots. Figure $1 \mathrm{~B}$ shows the crystal shape of two representative nanosized quantum dots. In this presentation, the practical limits of this method to quantify the size and shape of individual quantum dots will be discussed [4].

\section{References}

[1] W. Baumeister, and A.C. Steven, Trends Biochem. Sci, 25 (12) (2000) 624.

[2] J. R. Kremer, D. N. Mastronarde and J. R. McIntosh, J. Struc. Biol. 116 (1) (1996) 71.

[3] J. Frank et al., J. Struc. Biol. 116 (1) (1996) 190.

[4] This work was supported by the Petroleum Research Fund under grant number \# 42361-AC5
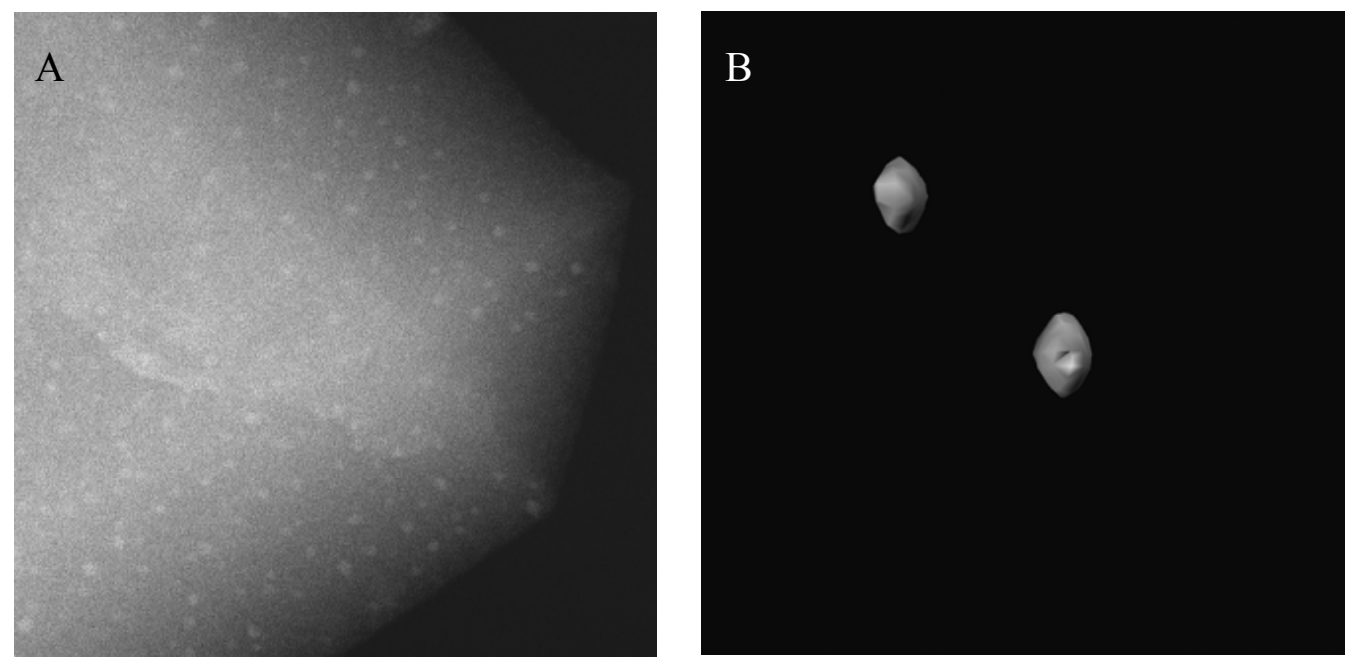

Fig. 1. A. The reconstructed 3D image of the Sn quantum dots in a silicon matrix. B. Crystal shape of two adjacent quantum dots. 